XXXI International Workshop on High Energy Physics:

Critical Points in the Modern Particle Physics

International Journal of Modern Physics: Conference Series

Vol. 47 (2018) 1860095 (5 pages)

(C) The Author(s)

DOI: $10.1142 / \mathrm{S} 2010194518600959$

\title{
Asymptotic Behavior and Critical Coupling in Scalar Yukawa Model
}

\author{
V. E. Rochev \\ NRC "Kurchatov Institute" — IHEP, \\ Protvino, Moscow Region 142281, Russian Federation \\ rochev@ihep.ru
}

Published 6 June 2018

\begin{abstract}
The solution of the equation for the pion propagator in the leading order of the $1 / N$ - expansion for a vector-matrix model with interaction $g\left(\phi_{a}\right)^{*} \phi_{b} \chi_{a b}$ in four dimensions shows a change of the asymptotic behavior in the deep Euclidean region in a vicinity of a certain critical value of the coupling constant.
\end{abstract}

Keywords: Quantum field theory; scalar Yukawa model; critical coupling.

In this report I will talk about one critical phenomenon found in a four-dimensional model.

I consider a vector-matrix model of the complex scalar field $\phi_{a}$ (pion) and real scalar mass-less field $\chi_{a b}$ (chion) with interaction $g\left(\phi_{a}\right)^{*} \phi_{b} \chi_{a b}$ in four dimensions $(a, b=1, \cdots, N)$. This model, known as well as a scalar Yukawa model, is used in nuclear physics as a simplified version of the Yukawa model without spin degrees of freedom, as well as an effective model of the interaction of scalar quarks (squarks). As the simplest model of the interaction of fields, this model often used as a prototype of more substantive theories to elaborate the various non-perturbative approaches in the quantum field theory.

The solution of the equation for the pion propagator in the leading order of the $1 / N$ - expansion shows a change of the asymptotic behavior in the deep Euclidean region in a vicinity of a certain critical value of the coupling constant. For small values of the coupling the propagator behaves as free, which is consistent with the wide-spread opinion about the dominance of perturbation theory for this super-renormalizable model. In the strong-coupling region, however, the asymptotic behavior changes dramatically - the propagator in the deep Euclidean region tend to some constant limit.

This is an Open Access article published by World Scientific Publishing Company. It is distributed under the terms of the Creative Commons Attribution 4.0 (CC-BY) License. Further distribution of this work is permitted, provided the original work is properly cited. 


\section{E. Rochev}

The similar change of asymptotic behavior was found also with solution of the system of Schwinger-Dyson equations in so-called two-particle approximation. ${ }^{1}$

The existence of a critical coupling constant in the scalar Yukawa model was noticed by practically all authors that have investigated this model using different methods. This critical constant is generally regarded as a limit on the coupling constant for a self-consistent description of the model by some method. In our approach, however, the self-consistent solution for propagator exists also in the strong-coupling region, and the existence of the critical coupling looks more like as a phase transition in accordance with the general definition of the phase transition as a sharp change of properties of the model with a smooth change of parameters.

The Lagrangian of the model is

$$
\mathcal{L}=-\partial_{\mu}\left(\phi_{a}\right)^{*} \partial_{\mu} \phi_{a}-m^{2}\left(\phi_{a}\right)^{*} \phi_{a}-\frac{1}{2}\left(\partial_{\mu} \chi_{a b}\right)^{2}+\frac{g}{\sqrt{N}}\left(\phi_{a}\right)^{*} \phi_{b} \chi_{a b}
$$

$(a, b=1, \cdots, N)$.

The technique of construction of the $1 / N$ - expansion for such models is wellknown $\left({ }^{2,3}\right)$, and the leading-order equation for the pion propagator $\Delta_{a b}(x)=$ $\delta_{a b} \Delta(x)$ is

$$
\Delta^{-1}(x)=\left(m^{2}-\partial^{2}\right) \delta(x)-g^{2} D_{c}(x) \Delta(x) .
$$

Here $x \in E_{4}$ and $D_{c}=-1 / \partial^{2}$.

At $N=1$ this equation coincides with the leading-order equation of the ladder expansion (see Refs. 4, 5), and in the language of Feynman diagrams it corresponds to the summation of ladder graphs.

To eliminate ultraviolet divergences in equation (2) it is necessary to introduce counter-terms of pion-field renormalization and mass. The normalization of the renormalized propagator $\Delta\left(p^{2}\right)$ at zero momentum leads to the renormalized equation in momentum space

$$
\Delta^{-1}\left(p^{2}\right)=m^{2}+p^{2}+\Sigma_{r}\left(p^{2}\right),
$$

where $\Sigma_{r}\left(p^{2}\right)=\Sigma\left(p^{2}\right)-\Sigma(0)-p^{2} \Sigma^{\prime}(0)$ is the renormalized mass operator, $m$ is the renormalized mass, and

$$
\Sigma\left(p^{2}\right)=-g^{2} \int \frac{d^{4} q}{(2 \pi)^{4}} D_{c}(p-q) \Delta(p) .
$$

After angle integrations we obtain the integral equation:

$$
\Delta^{-1}\left(p^{2}\right)=m^{2}+(1-\lambda) p^{2}+2 \lambda m^{2} \int_{0}^{p^{2}} \Delta\left(q^{2}\right)\left(1-\frac{q^{2}}{p^{2}}\right) d q^{2}
$$

where

$$
\lambda \equiv \frac{g^{2}}{32 \pi^{2} m^{2}}
$$

- dimensionless coupling. 
This equation can be reduced to the non-linear differential equation

$$
\frac{d^{2}}{\left(d p^{2}\right)^{2}}\left(p^{2} \Delta^{-1}\left(p^{2}\right)\right)=2(1-\lambda)+2 \lambda m^{2} \Delta\left(p^{2}\right) .
$$

We shall look for the positive solutions $\left(\Delta^{-1}(p)>0\right)$ of the equation for the propagator in the region of large momenta. Negative solutions necessarily contain Landau singularities and are therefore physically unacceptable.

In the weak-coupling region $\lambda<1$ the asymptotic solution at large $p^{2}$ is

$$
\Delta(p)=\frac{1}{(1-\lambda) p^{2}}\left(1-\frac{2 \lambda m^{2}}{(1-\lambda)^{2}} \frac{\ln p^{2} / m^{2}}{p^{2}}+O\left(1 / p^{2}\right)\right)
$$

This asymptotic solution is self-consistent in region $\lambda<1$ and corresponds to the asymptotically-free behavior of propagator.

At $\lambda=1$ the equation for the propagator can be reduced to singular EmdenFowler equation:

$$
\ddot{y}=2 t y^{-1} \text {. }
$$

Here

$$
y=\frac{p^{2}}{\left(m^{2}\right)^{2}} \Delta^{-1}(p), \quad t=\frac{p^{2}}{m^{2}} .
$$

According to this, the asymptotic behavior of propagators in the critical point $\lambda=1$ has the form

$$
\Delta(p)=\sqrt{\frac{3}{8 m^{2} p^{2}}}\left(1+O\left(1 / p^{2}\right)\right)
$$

at large $p^{2}$ and drastically differs from the asymptotically-free behavior in the weakcoupling region.

In the strong-coupling region $\lambda>1$, the equation for the propagator has the positive approximate solution

$$
\Delta^{-1}\left(p^{2}\right) \simeq \frac{\lambda m^{2}}{\lambda-1}\left[1-\frac{1}{\lambda-1} \sqrt{\frac{m^{2}}{2 \lambda p^{2}}} J_{1}\left(2(\lambda-1) \sqrt{\frac{2 p^{2}}{\lambda m^{2}}}\right)\right]
$$

(here $J_{1}$ is the Bessel function), which has the right normalization in zero $\left(\Delta^{-1}(0)=\right.$ $m^{2}$ ) and the constant asymptotic behavior

$$
\Delta(\infty)=\frac{\lambda-1}{\lambda m^{2}}
$$

At $\lambda=1$ the asymptotic behavior of the propogator $(1 / p)$ is a medium among the free behavior $1 / p^{2}$ at $\lambda<1$ and the constant-type behavior in strong-coupling region $\lambda>1$. A sharp change of asymptotic behavior in the vicinity of the critical value is a behavior that is characteristic for a phase transition. This phase transition is not associated with a symmetry breaking, and in this sense is similar on the phase transition "gas-liquid". The weak coupling region can be roughly classified as the gaseous phase and the strong coupling region (where a kind of localization 
of correlators exists, see below) - to the liquid. This analogy, of course, is a quite shallow. A type and characteristics of this phase transition can be defined as the result of a more detailed study.

This phase transition is similiar, in a sense, to the phenomenon of re-arrangement of physical vacuum in the strong external field (see Ref. 6 and references therein).

The pion propagator in the strong-coupling region asymptotically approaches to a constant. It is not something unexpected, if we remember the well-known conception of the static ultra-local approximation. In this approximation, all the Green functions are combinations of $\delta$-functions in the coordinate space that are constants in momentum space. Of course, this approximation is very difficult for a physical interpretation. Nevertheless, it can be considered as a starting point for an expansion in inverse powers of the coupling constant, i.e., as a leading approximation of the strong-coupling expansion (see, e.g., Ref. 7).

Note, that in contrast to the ultra-local approximation, our solution are free from the interpretation problem, since for the small momenta it has the quite traditional pole behavior.

Strong-coupling propagator (6) has very interesting shell structure in the fourdimensional Euclidean $\mathrm{x}$-space. Doing Fourier transform of (6) we obtain:

$$
\Delta^{-1}(x)=\int \frac{d^{4} p}{(2 \pi)^{4}} e^{-i p x} \Delta^{-1}(p)=\frac{\lambda m^{2}}{\lambda-1}\left(\delta^{4}(x)-\frac{m^{2}}{8 \pi^{2}(\lambda-1)^{2}} \delta\left(x^{2}-x_{0}^{2}\right)\right),
$$

where the quantity

$$
x_{0}=(\lambda-1) \sqrt{\frac{8}{\lambda m^{2}}} \sim \lambda_{\text {compton }}
$$

can be considered as the size of the pion localization region (a "radius" of the pion).

This radius increases as the coupling increases, which is very natural for the strong-coupling regime.

- The main result is finding of the change of asymptotic behavior in the scalar Yukawa model. The equation for the pion propagator in the leading order of $1 / N-$ expansion has self-consistent positive solutions in the Euclidean region not only in the weak-coupling region, (where a dominance of the perturbation theory in this model is obvious), but also in the strong-coupling region. Of interest is the further study of this critical phenomenon, in particular, the investigations of amplitudes in this model and the search for analogs in other, more realistic models.

\section{References}

1. V.E. Rochev, J. Phys. A: Math. Theor. A46, 185401 (2013).

2. G. 't Hooft, Nucl. Physics B 75, 461 (1974).

3. A.A. Slavnov, Theor. Math. Phys. 51307 (1982).

4. V.E. Rochev, Phys. Atom. Nucl., 78, 443 (2015). [Yad. Fiz., 78, 475.] 
5. V.E. Rochev, J. Mod. Phys. 7, 899 (2016).

6. W. Greiner, B. Müller, J. Rafelski, Quantum Electrodynamics of Strong Fields (Springer-Verlag, 1985).

7. R.J. Rivers, Path Integral Methods in Quantum Field Theory (Cambridqe Univ. Press, 1987). 